\title{
Development of the Needle Insertion Robot for Percutaneous Vertebroplasty
}

\author{
S. Onogi ${ }^{1}$, K. Morimoto ${ }^{1}$, I. Sakuma ${ }^{1}$, Y. Nakajima ${ }^{2}$, T. Koyama ${ }^{3}$, \\ N. Sugano ${ }^{3}$, Y. Tamura ${ }^{4}$, S. Yonenobu ${ }^{4}$, and Y. Momoi ${ }^{5}$ \\ ${ }^{1}$ Graduate School of Frontier Sciences, The University of Tokyo, Japan \\ 2 Intelligent Modeling Laboratory, the Univeristy of Tokyo, Japan \\ 3 Graduate School of Medicine, Osaka University, Japan \\ 4 Osaka Minami Medical Center, Japan \\ 5 Hitachi Ltd
}

\begin{abstract}
Percutaneous Vertebroplasty (PVP) is an effective and less invasive medical treatment for vertebral osteoporotic compression fractures. However, this operative procedure is quite difficult because an arcus vertebra, which is narrow, is needled with accuracy, and an operator's hand is exposed to X-ray continuously. We have developed a needle insertion robot for Percutaneous Vertebroplasty. Its experimental evaluation on the basic performance of the system and needle insertion accuracy are presented. A needle insertion robot is developed for PVP. This robot can puncture with accuracy and an operator does not need to be exposed to X-ray. The mechanism of the robot is compact in size $(350 \mathrm{~mm} \times \mathrm{D} 400 \mathrm{~mm} \times \mathrm{H} 270 \mathrm{~mm}$, weight: $15 \mathrm{~kg})$ so that the robot system can be inserted in the space between C-arm and the patient on the operating table. The robot system is controlled by the surgical navigation system where the appropriate needle trajectory is planned based on pre-operative three-dimensional CT images. The needle holding part of the robot is X-ray lucent so that the needle insertion process can be monitored by fluoroscopy. The position of the needle during insertion process can be continuously monitored. In vitro evaluation of the system showed that average position and orientation errors were less than $1.0 \mathrm{~mm}$ and 1.0 degree respectively. Experimental results showed that the safety mechanism called mechanical fuse released the needle holding disk properly when excessive force was applied to the needle. These experimental results demonstrated that the developed system has the satisfactory basic performance as needle insertion robot for PVP.
\end{abstract}

\section{Introduction}

Percutaneous Vertebroplasty(PVP) is an effective treatment for vertebral osteoporotic compression fractures (Figure 11). In this technique, the surgeon inserts one or two bone biopsy needles into fractured vertebral body, and injects semiliquid plastic cement called bone cement into the vertebral body through the needle. After injection the bone cement hardens, the vertebra is stabilized. In 
this treatment technique, it is one of the most important procedure that the surgeon inserts needle into vertebra precisely. Because the spinal cord and nerves exist through the vertebra, if the surgeon injures nerves by the needle, it will cause critical accidents such as a partial paralysis of the patients. The surgeon must insert needles along appropriate trajectory that locates in a narrow space of pedicle of arch of vertebra. Thus, surgeons must have considerably high skill and experiences in order to control the position of needle. When the needle is inserted percutaneously, the surgeon uses X-ray fluoroscopy to confirm the position of needle resulting in continuous exposure of surgeon's hand to X-ray. A new engineering assistance is required to improve the reliability, accuracy, and safety of this procedure.

In order to improve the abovementioned subject, we have developed a needle insertion robot for PVP (Figure 2) 1. Cleary et al. developed needle insertion robot for nerve and facet blocks under X-ray fluoroscopy 2. Compared with robot for nerve and facet blocks, the robot for PVP must generate larger insertion force to make the needle penetrate cortical bone of vertbra. On the other hand, the size of the robot must be compact. In

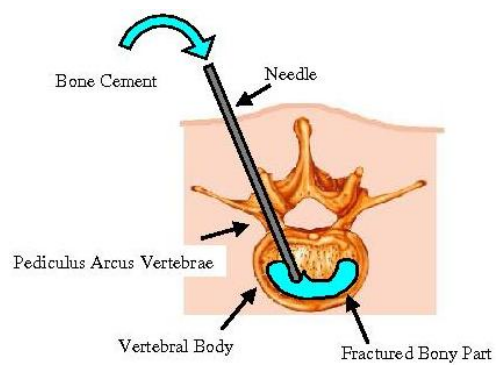

Fig. 1. Percutaneous Vertebroplasty this report, design of the developed needle insertion robot for percutaneous vertebroplasty, its experimental evaluation on the basic performance of the system and needle insertion accuracy are presented. The positioning accuracy of the robot itself was evaluated and the safety mechanism in case of excessive applied force was also tested. Finally, accuracy of needle insertion of the robot under image guidance was evaluated using a vertebra model.

\section{Materials and Methods}

\section{Design of the Needle Insertion Robot}

The developed robot has the following features:

1. The robot is rigid enough to generate required needle insertion force.

2. The robot is compact so that the robot system (needle positioning mechanism, needle insertion and rotation mechanism) can be inserted in the space between $\mathrm{C}$-arm and the patient on the operating table. We have also developed an X-ray lucent operating table made of carbon reinforced fiber materials (Mizuho Ltd., Japan).

3. The needle holding part of the robot is X-ray lucent so that the needle insertion process can be monitored by fluoroscopy (Figure 3).

4. The position and orientation of the needle can be adjusted with five degrees of freedom in three-dimensional space. 
5. The robot system is controlled by the surgical navigation system where the appropriate needle trajectory is planned based on pre-operative three dimensional CT images.

6. The safety mechanism that avoid injury of the patient by the needle when excessive force is applied to the needle due to malfunction of the system.

The needle insertion robot is shown in Figure 2. The robot consists of three parts: 1) Rough positioning mechanism, 2) Accurate positioning mechanism, 3) Puncture mechanism. The rough positioning mechanism does not have any actuator. It has only electro-magnetic brake to fix two joints. (X, and $\mathrm{Y}$ in Figure 4). It positions the accurate positioning mechanism and puncture mechanism in two-dimensional plane parallel to the operation bed surface. One actuated translational positioning mechanism is used to position the mechanism in $\mathrm{z}$ direction shown in Figure 4 .

The accurate positioning mechanism has four degrees of freedom for determination of orientation and position of the puncture mechanism: two DOF for to perpendicular translational motions $( \pm 10 \mathrm{~mm}$ in $\mathrm{s}[1]$ and $\mathrm{s}[2]$ direction shown in Figure 4) and two DOF for rotating motions around two axis intersecting with each other at right angle $( \pm 30$ degrees in $\alpha$ and \pm 5 degrees in $\beta$ shown in

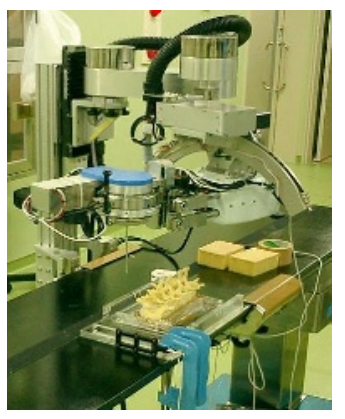

Fig. 2. Developed Needle Insertion Robot

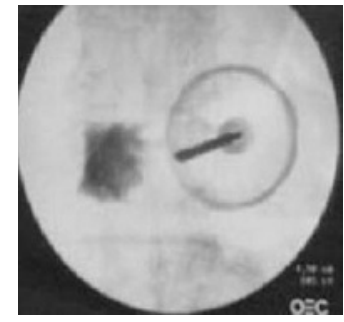

Fig. 3. C-arm X-ray image of the robot which has radiolucent

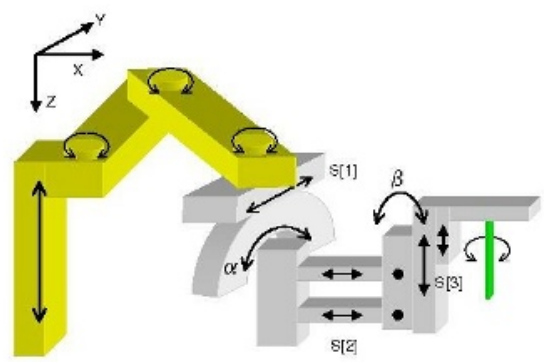

Fig. 4. Robot Mechanism 
Figure (4). R-guide was used to realize the rotation for $\alpha$ axis, and remote center of motion mechanism consisting of two linear actuation mechanism was used to reduce the thickness of the mechanism 3 .

The puncture mechanism inserts the needle into the patients (shown as s[3] in Figure (4) and rotates the needle in reciprocal manner with amplitude of 120 degrees. The stroke and resolution of the needle insertion mechanism are 110 $\mathrm{mm}$ and $\pm 0.2 \mathrm{~mm}$ respectively (Figure 4). The holder is a plastic disk fixed to cylindrical part made of stainless steel that is rotated by a DC motor. Inner diameter of the cylinder is $52 \mathrm{~mm}$. We can observe the position of needle by intra operative X-ray fluoroscopy through the cylinder. Surgeon can monitor the position of the tip of the needle during needle insertion process. It also has a force/torque sensor to measure the force applied on the robot during needle insertion. It is reported that the axial force during needle insertion to human vertebra preserved under formalin fixation. And it is reported that the forces did not exceed $25 \mathrm{~N}$ when feed rate of the needle was $0.05-0.5 \mathrm{~mm} / \mathrm{s}$. We designed the needle insertion mechanism to generate up to $60 \mathrm{~N}$ of axial force.

This robot has safety mechanism, called "Mechanical Fuse" (Figure 5). The needle was fixed on the disk plate. The holder grasps the disk with four contacting parts supported by springs as shown in Figure 6. When unexpected excessive force is applied to a needle, the disk comes off from the holder to avoid possible damage
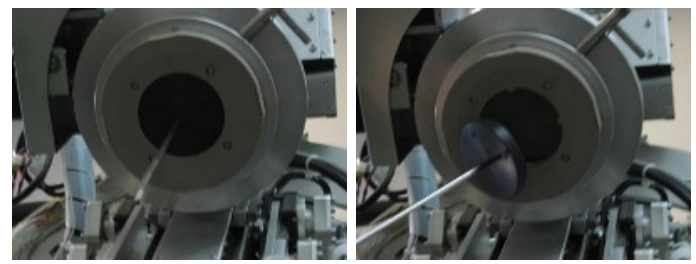

Fig. 5. Left: Normal setting, Right: Situation of Needle comes off by Mechanical Fuse to the patient.

The entire mechanism was designed to be fixed to the operating table. The size of the entire mechanism was $350 \mathrm{~mm} \times \mathrm{D} 400 \mathrm{~mm} \times \mathrm{H} 270 \mathrm{~mm}$, and its weight was $15 \mathrm{~kg}$. It can be inserted in the space between C-arm of the patient on the operating table as shown in Figure 7 .

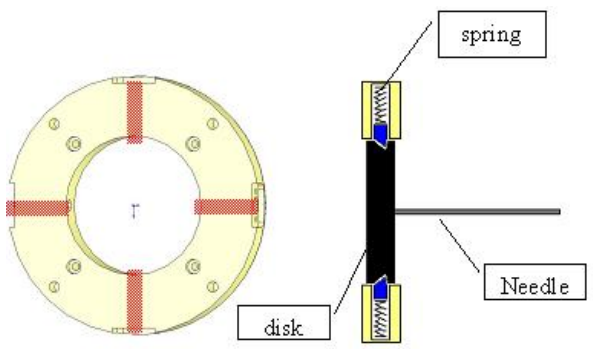

Fig. 6. Mechanism of the Mechanical Fuse

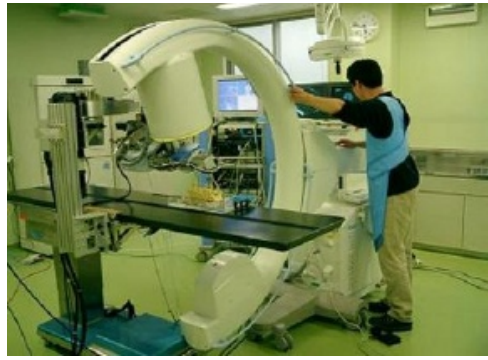

Fig. 7. The robot can be installed between a patient and C-Arm XRay Equipment 


\section{Description of Control System}

Next, control system is shown in Figure 8 . The system consists of the following three devices: 1) Needle Insertion Robot, 2) Navigation System(4), 3) Optical Position Sensor (Optotrak, NDI, Canada). The robot is connected the navigation system by LAN cable (TCP/IP). And the navigation system is connected with an optical Position Sensor by serial cable (RS232C). The navigation system sends the position and orientation data to the robot. The robot drives the target position and orientation using this data by software.

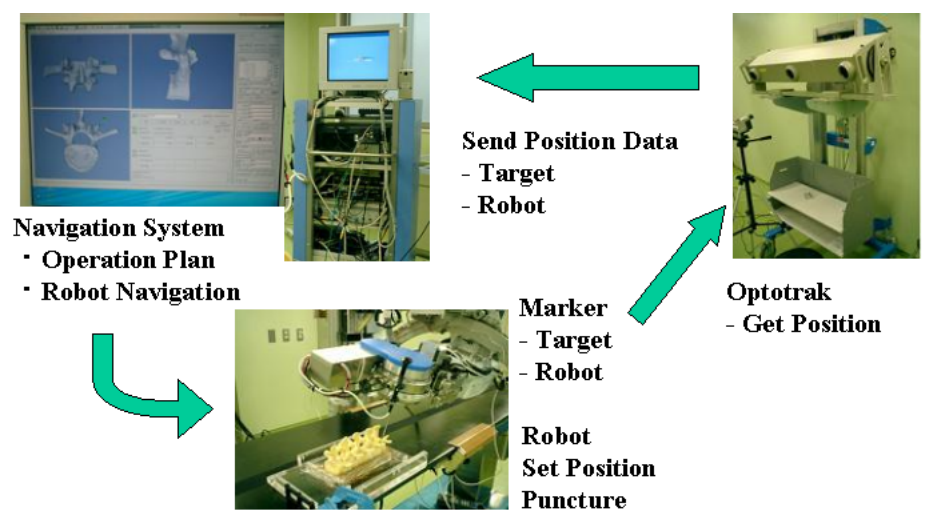

Fig. 8. Total System

\section{Evaluation of Needle Positioning Accuracy}

In this study, three experiments were conducted. First experiment is accuracy evaluation of the robot positioning ability. Target values in robot coordinate system were input to the robot and errors between target values and real values which were measured with a position sensor (Polaris, NDI, Canada) placed at the needle insertion mechanism. The errors were evaluated for various points and orientations in the range of motion of the robot.

Second experiment was evaluation of the Mechanical Fuse. The needle was hold by a material-testing instrument with force sensor. The force was applied along the needle and from the direction perpendicular to the needle. The force applied to the needle when the disk came off from the holder was recorded.

Third experiment is evaluation of needle insertion accuracy as a entire system including positioning errors due to needle insertion robot, surgical navigation system, and optical position sensor using a vertebra model (Sawbones, Pacific Research Laboratories, USA). (Figure 9). Three dimensional computer model of the vertebra model was obtained based on its

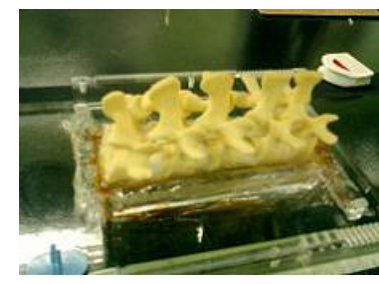

Fig. 9. Polyurethane Vertebra Phantom 
$\mathrm{CT}$ data. The surface registration was used for surgical navigation $[5|6| 7 \mid 8]$. The total errors between target values and determinate needle positions were measured by comparing the planned positions of the needle insertion mechanism and measured position data obtained by the optical position sensor. CT data after the needle insertion was also obtained to identify the difference between the actual needle trajectory and needle insertion plan for one case of the experiments.

The operation with this robot has three stages. At first stage, rough position is set manually. Second, accurate position is set automatically by interaction with the navigation system. Third, needle is inserted to a vertebra.

\section{Results}

\section{Accuracy Evaluation of the Robot Positioning}

Accuracy evaluation is performed on each axis. Result is shown in Table 1 All axes are satisfied requested specifications. However, errors of axis $\mathrm{X}$ and $\mathrm{Z}$ are somewhat large.

Next, target values in a robot coordinate system are inputted the robot. Result is shown in Table 2, In this case, all axes are satisfied requested specifications, too.

\section{Evaluation of the Mechanical Fuse}

Force along the insertion direction larger than $50 \mathrm{~N}$ made the disk came off the holder. Needle holding disk came off when force larger than $3 \mathrm{~N}$ was applied at the tip of the needle perpendicular to the needle direction. To simulate the possible situation in clinical setting, position of the vertebra model after initial needle insertion was shifted purposely by manual. The needle came off by mechanical fuse successfully. When a vertebra model is moved suddenly, the needle came off from the robot, too.

\section{Evaluation of Puncture in a Vertebra Model}

The target value is set by the navigation system with CT image of pre-operation. The position and orientation errors before and after contacting the model were shown in Table 3. The contact of the needle was detected by the force sensor signals from the system.

$\mathrm{X}$-ray images of one vertebra model used in the experiments were obtained by $\mathrm{CT}$ to identify the position of holes created by the needle. The position and trajectory of the needle insertion was evaluated as the center of the respected

Table 1. Accuracy evaluation result of each axis $(n=)$

\begin{tabular}{|l|l|l|l|l|}
\hline $\mathrm{X}(\mathrm{n}=15)$ & $\mathrm{Y}(\mathrm{n}=15)$ & $\mathrm{Z}(\mathrm{n}=12)$ & $\alpha(\mathrm{n}=36)$ & $\beta(\mathrm{n}=27)$ \\
\hline $0.54 \mathrm{~mm}$ & $0.09 \mathrm{~mm}$ & $0.80 \mathrm{~mm}$ & $0.25 \mathrm{deg}$ & $0.41 \mathrm{deg}$ \\
\hline
\end{tabular}


Table 2. Accuracy evaluation result of multiple axes

\begin{tabular}{|c||c|c|c|}
\hline & Ave. & SD & Max. Err. \\
\hline $\mathrm{X}[\mathrm{mm}](\mathrm{n}=675)$ & -0.437 & 0.325 & -1.197 \\
\hline $\mathrm{Y}[\mathrm{mm}](\mathrm{n}=900)$ & -0.158 & 0.197 & -0.675 \\
\hline $\mathrm{Z}[\mathrm{mm}](\mathrm{n}=1428)$ & -0.540 & 0.361 & -1.476 \\
\hline$\alpha[\mathrm{deg}](\mathrm{n}=2448)$ & 0.122 & 0.0448 & 0.230 \\
\hline$\beta[\mathrm{deg}](\mathrm{n}=279)$ & 0.138 & 0.0661 & 0.364 \\
\hline
\end{tabular}

Table 3. Result of Puncture Experiment

\begin{tabular}{|c|c|c|c|c|}
\hline & & Average Error \pm SD & Maximum Error & Minimum Error \\
\hline $\begin{array}{c}\text { Pre-Puncture } \\
(\mathrm{n}=10)\end{array}$ & Position $[\mathrm{mm}]$ & $0.80 \pm 0.29$ & 1.13 & 0.31 \\
\cline { 2 - 5 } & Orientation $[\mathrm{deg}]$ & $0.06 \pm 0.08$ & 0.21 & 0.00 \\
\hline $\begin{array}{c}\text { Contact } \\
(\mathrm{n}=8)\end{array}$ & Position $[\mathrm{mm}]$ & $0.81 \pm 0.40$ & 1.51 & 0.41 \\
\cline { 2 - 5 } & Orientation $[\mathrm{deg}]$ & $0.20 \pm 0.26$ & 0.80 & 0.03 \\
\hline
\end{tabular}

volume due to needle insertion. The difference between the planned position and the entry point of the needle at the surface was $0.21 \mathrm{~mm}$ and the orientation error was $0.9 \mathrm{deg}$.

\section{Discussion}

The developed robot is compact enough to be set in the space between C-arm and operating table, while being able to generate required force for needle insertion to vertebra. It can insert $10 \mathrm{G}$ needle into porcine vertebra sample with surrounding tissue and skin (data not shown.) Thus, it can generate enough force for PVP.

In accuracy evaluation, the robot is satisfied requested specifications (error less than $1 \mathrm{~mm}$ ). The reason that errors of axis $\mathrm{X}$ and $\mathrm{Z}$ are somewhat large is mechanism of $\mathrm{X}$-axis for small size. We used a remote center of motion mechanism consisting of two linear actuation mechanisms to reduce the thickness of the mechanism placed in the space between the C-arm of the fluoroscopy and operating table. This deteriorates positioning accuracy of the robot. However, since required positioning accuracy was satisfied as a whole. The system has enough positioning accuracy for PVP.

The Mechanical Fuse functioned as designed. For further validation of the mechanical fuse, experiments simulating possible disturbances observed in actual clinical situations to confirm the safety of the system. In addition to the mechanical safety measures, we have to develop the software to stop the system when the abnormal needle force is detected in the embedded force sensors in the system.

In total system error evaluation experiments, average of error is satisfied requested specifications (position error less than $1 \mathrm{~mm}$ and orientation error less than $1 \mathrm{deg}$ ). However, there was a case of the error exceeding required 
specification. We have to investigate the possible causes of errors to reduce the total positioning accuracy. Another possible factor not evaluated in the present study is the slip of the needle tip at the contact to the cortical bone. When the surface of the vertebra is inclined, the needle may slip from the appropriate insertion position. Thus the appropriate needle insertion plan must be designed to reduce the possibility of needle slip based on the geometrical information of the vertebra. Although the system can be inserted into the space between the Carm and patient on the operating table, the system must be further miniaturized for ease of operation for easier setting and operation. We will analyze the cause of errors and optimize the mechanical design.

\section{Conclusion}

We have developed a needle insertion robot for Percutaneous Vertebroplasty. Its experimental evaluation on the basic performance of the system and needle insertion accuracy is presented. This robot can puncture with accurate and an operator does not need to be exposed to X-ray. The mechanism of the robot is compact in size $(350 \mathrm{~mm} \times \mathrm{D} 400 \mathrm{~mm} \times \mathrm{H} 270 \mathrm{~mm}$, weight: $15 \mathrm{~kg})$ so that the robot system can be inserted in the space between $\mathrm{C}$-arm of the patient on the operating table. The position and orientation of the needle can be adjusted with five degrees of freedom in three-dimensional space. The robot system is controlled by the surgical navigation system where the appropriate needle trajectory is planned based on pre-operative three-dimensional CT images. The needle holding part of the robot is X-ray lucent so that the needle insertion process can be monitored by fluoroscopy. The position of the needle during insertion process can be continuously monitored. In vitro evaluation of the system showed that average positioning and orientation errors were less than $1.0 \mathrm{~mm}$ and $1.0 \mathrm{de}-$ gree respectively. Experimental results showed that the safety mechanism called mechanical fuse released the needle holding disk properly when excessive force was applied to the needle. These experimental results demonstrated that the developed system has the satisfactory basic performance as needle insertion robot for PVP.

Acknowledgement. This study was partly supported by "Research for the Future Program JSPS-RFTF 99I00904"

\section{References}

1. Matsumiya, K.: Proc. miccai 2003. Medical Image Computing and ComputerAssisted Intervention (2003) 271-278

2. Cleary, K., Stoianovici, D., Patriciu, A., Mazilu, D., Lindisch, D., Watson, V.: Robotically assisted nerve and facet blocks. Academic Radiology (2002) 821-825

3. Kim, D., Kobayashi, E., Dohi, T., Sakuma, I.: A new, compact mr-compatible surgical manipulator for minimally invasive liver surgery. Lecture Notes in Computer Science 2488 (2002) 99-106 
4. Nakajima, Y., Yamamoto, H., Sato, Y., Sugano, N., Momoi, Y., Sasama, T., Koyama, T., Tamura, Y., Yonenobu, K., Sakuma, I., Yoshikawa, H., Ochi, T., Tamura, S.: Available range analysis of laser guidance system and its application to monolithic integration with optical tracker. CARS 2004, Chicago, USA (2004-06)

5. Besl, P.J., Mckay, N.D.: A method for registration of 3-d shapes. IEEE Trans. Pattern Anal. Machin. Intell. 14 (1992) 239-256

6. Simon, D., Hebert, M., Kanade, T.: Techniques for fast and accurate intrasurgical registration. The Journal of Image Guided Surgery 1 (1995) 17-29

7. C.R.Maurer, J.Maciunas, J.M.Fitzpatrick: Registration of head ct images to physical space using a weighted combination of points and surfaces. IEEE Trans. Med. Imag. 17 (1998) 753-761

8. R.Bächler, H.Bunke, L.P.Nolte: Restricted surface matching-numerical optimization and technical evaluation. Computer Aided Surgery 6 (2001) 143-152 